\title{
Stratégies patronales de rénovation du dialogue social dans les années 1970
}

Discrimination de l'action syndicale à la SNIAS de Toulouse

Employers' Strategies for Changing the Relationship with Trade Unions.

Discrimination and the SNIAS in Toulouse in the Early 1970 s

Clair Juilliet

\section{(2) OpenEdition}

\section{Journals}

Édition électronique

URL : http://journals.openedition.org/travailemploi/6917

DOI : 10.4000/travailemploi.6917

ISSN : 1775-416X

Éditeur

DARES - Ministère du Travail

\section{Édition imprimée}

Date de publication : 1 janvier 2016

Pagination : 147-172

ISSN : 0224-4365

\section{Référence électronique}

Clair Juilliet, «Stratégies patronales de rénovation du dialogue social dans les années 1970 », Travail et Emploi [En ligne], 145 | janvier-mars 2016, mis en ligne le 01 janvier 2018, consulté le 21 avril 2019. URL : http://journals.openedition.org/travailemploi/6917; DOI : 10.4000/travailemploi.6917 


\title{
Stratégies patronales de rénovation du dialogue social dans les années 1970
}

\author{
Discrimination de l'action syndicale à la SNIAS de Toulouse
}

\author{
Clair Juilliet
}

\begin{abstract}
Au début des années 1970, la direction de l'établissement toulousain de la Société nationale industrielle aérospatiale (SNIAS) souhaite procéder à une transformation des pratiques en matière de relations sociales. Pour ce faire, elle recourt à un certain nombre de dispositifs visant à modifier le rapport de forces avec les syndicats et à marginaliser le syndicalisme CGT et CFDT au profit de l'approche réformiste développée par la CGT-FO, la CGC ou la CFTC, organisations plus favorables à la politique contractuelle portée par l'entreprise. Pour permettre cette rénovation sociale, la direction s'appuie sur des procédés répressifs et/ou discriminants qui ont pour conséquence d'affaiblir rapidement et durablement l'axe revendicatif au sein de l'établissement. Dans cet article, nous décrivons les stratégies directes et indirectes utilisées par la direction pour parvenir à un apaisement durable des relations sociales dans les usines.
\end{abstract}

$\mathrm{D}$ ans les années 1960, l'industrie aéronautique, secteur stratégique, connaît des évolutions majeures en matière économique, sociale ou encore technique (CARLIER, 1983). Sud-Aviation, principale Société nationale de constructions aéronautiques (SNCA), développe son outil de production, en particulier grâce au succès relatif rencontré par les programmes Caravelle et Alouette et aux espoirs placés dans la réussite du Concorde. Cette situation s'accompagne néanmoins d'importantes disparités sociales entre les établissements et d'une micro-conflictualité permanente, aux visages multiples (MoREL, 1994). La réorganisation qui intervient au $1^{\mathrm{er}}$ janvier 1970 avec la fusion de Sud-Aviation et de Nord-Aviation au sein de la Société nationale industrielle aérospatiale (SNIAS), le développement de la coopération européenne autour du programme Airbus et le passage progressif d'une logique d'arsenal à une

\footnotetext{
* Framespa (France, Amériques, Espagne - sociétés, pouvoirs, acteurs ; UMR 5136, université Toulouse-Jean Jaurès) ; labEx Structurations des mondes sociaux (SMS), université de Toulouse ; clairjuilliet@gmail.com.
} 
logique commerciale (KECHIDI, TALBOT, 2006) incitent la direction à entreprendre une réforme du dialogue social et de la politique de gestion des ressources humaines. Cette réforme a notamment pour but d'augmenter la productivité du travail et d'affaiblir le contre-pouvoir syndical. Ce faisant, la direction souhaite « pacifier » l'établissement où est réalisé l'assemblage des avions, c'est-à-dire le site de Toulouse ${ }^{1}$, afin qu'y soit assurée la continuité du travail nécessaire au développement de l'entreprise.

Comme nous allons le montrer, la direction a recours pour ce faire à un certain nombre de méthodes visant à modifier le rapport de forces avec les syndicats et à marginaliser la Confédération générale du travail (CGT) et la Confédération française démocratique du travail (CFDT) : ainsi, elle promeut un syndicalisme CGT-FO ${ }^{2}$ et Confédération générale des cadres (CGC) (puis Confédération française des travailleurs chrétiens [CFTC]), plus favorable à la politique contractuelle; et elle utilise un certain nombre de procédés directement répressifs et discriminants à l'égard de la CGT et de la CFDT. Cette volonté de reprise en main des usines perturbe, puis affaiblit les organisations qui ne s'inscrivent pas dans la démarche de rénovation du dialogue social. Elles ne peuvent plus peser sur les décisions économiques et sociales et sur les perspectives en matière industrielle. Quelles sont précisément ces stratégies mises en œuvre par la direction pour affaiblir durablement le syndicalisme CGT et, dans une moindre mesure, CFDT ? Elles nous semblent s'apparenter à une discrimination de l'action syndicale si l'on donne à la notion une acception large, où celle-ci peut être directe ou indirecte, individuelle ou collective, formelle ou informelle, préventive ou répressive, dans ou hors du cadre juridique, etc. La discrimination peut de fait être considérée comme un phénomène complexe et protéiforme, aux ramifications multiples (MERCAT-BRUNS, 2011).

Cette réforme à la tonalité discriminatoire s'inscrit dans un contexte économique, politique et social particulier. Elle s'appuie sur un syndicalisme dit réformiste, susceptible de représenter une alternative crédible pour les travailleurs et incarné dans l'établissement par FO, syndicat qui entend contester le leadership cégétiste en vigueur depuis la fin des années 1940. La réforme engagée dans l'entreprise, comme plus largement les orientations patronales de l'époque, vise précisément à soutenir cet axe réformiste, comme le montrent deux documents « confidentiels » dévoilés par les militants CGT de l'usine de Saint-Nazaire (BELLIOT et al., 2012). Daté de novembre 1976, le premier document est une note interne au Conseil national du patronat français (CNPF) détaillant les difficultés rencontrées par FO et la CGC, qui sont confrontées à « l'attitude de chefs d'entreprise trop peu enclins à négocier avec les syndicalistes libres ${ }^{3}$ parce qu'ils estiment insuffisamment rentable de signer un accord avec eux ${ }^{4} »$. Le CNPF y conseille aux dirigeants de s'appuyer sur les syndicats réformistes dans

1. L'établissement compte trois usines principales : Blagnac, Saint-Martin-du-Touch et Saint-Éloi.

2. Syndicat également désigné couramment par le sigle FO (Force ouvrière).

3. L'expression désigne les syndicats réformistes, en particulier FO et la CGC.

4. « Note confidentielle du CNPF en faveur du syndicalisme réformiste, novembre 1976 », cité dans BELLIOT et al., 2012, pp. 50-53. 
un contexte de ralentissement de la politique contractuelle (plan Barre) : « On ne peut pas courir le risque de voir des syndicalistes libres [...] tentés de faire front commun avec la CGT et la CFDT. [...] Il convient donc de renforcer et de revaloriser FO [et de] favoriser le syndicalisme cadre qui apparaît indispensable pour combattre la force d'attraction qu'exerce la CGT sur certains cadres séduits par un modèle marxiste de société ${ }^{5} »$. Le syndicat patronal affirme en conclusion que « les chefs d'entreprise ont donc une responsabilité à assumer vis-à-vis du syndicalisme réformiste ${ }^{6} »$. La seconde note, de deux pages et demi, a été produite par la direction générale de la SNIAS en 1978. Elle revient longuement sur l'évolution des mesures à appliquer « en faveur de FO, CFTC et CGC ». Elle demande à :

«La hiérarchie de cesser les pressions directes qu'elle exerce sur le personnel en faveur de l'adhésion aux organisations syndicales FO, CFTC, CGC [...]. Ces mesures, jusqu'à présent efficaces, ont atteint leurs limites [...] : le personnel malléable a été touché ; les promesses d'augmentation et de promotion individuelle ne peuvent être généralisées à tous ; le maintien des pressions directes aboutirait à l'assimilation de ces organisations à la Direction, et à l'intervention des pouvoirs publics. Une attitude, plus subtile, s'impose ; le but essentiel [...] est de faciliter et de valoriser [leur] action. »

( « Note très confidentielle - SNIAS 1978 », cité dans BELLIot et al. [2012], pp. 54-56)

L'objectif de l'article est de présenter de façon précise les principaux aspects de cette réforme des relations sociales au moyen d'une analyse sociohistorique. Par le croisement des éléments factuels et points de vue qu'ils permettent, le dépouillement de sources diverses et les entretiens conduits avec des acteurs de l'époque (voir encadré) démontrent l'existence d'un répertoire d'actions visant à combattre une forme de syndicalisme au profit d'une autre, par l'emploi de dispositifs susceptibles d'influencer la négociation collective. Sans trop anticiper sur nos résultats, nous verrons que cette tentative réussie s'appuie, à court et moyen termes, sur des stratégies ponctuelles et continues : discrimination salariale, contrôle de l'embauche et blocage de carrière, mobilisation de la hiérarchie visent le syndicalisme CGT et CFDT de manière directe ; l'instauration d'une politique contractuelle sur les bases proposées par la direction, le favoritisme accordé aux organisations qui y participent, ou encore l'application plus stricte des lois portant sur les institutions représentatives du personnel (IRP) les touchent de manière indirecte. Toutes traduisent des discriminations individuelles et collectives (GARNER-MOYER, 2003), qui s'intègrent dans une stratégie de long terme plus globale d'écartement des syndicats développant une approche conflictuelle de la négociation.

5. « Note confidentielle du CNPF en faveur du syndicalisme réformiste, novembre 1976 », doc. cité.

6. Ibid. 


\section{ENCADRÉ}

\section{Sources et méthode d'analyse}

Les archives de l'établissement toulousain ont été en partie détruites, perdues, ou ont été rendues inaccessibles suite à la privatisation de l'entreprise en 1999. Plusieurs fonds ont été mobilisés pour combler cette carence, croiser les sources, les mettre à distance et donner un aperçu le plus fidèle possible de la situation. Parmi ceux-ci :

- les archives de l'Institut d'histoire sociale CGT 31 (IDHS 31) rassemblent un nombre important de tracts (de tous syndicats), de journaux d'entreprise, d'interviews, de rapports et d'analyses sur l'industrie aéronautique, etc. Les documents sur l'industrie aéronautique qui y sont conservés couvrent la période qui va de 1917 à nos jours ;

- les archives de l'union départementale (UD) FO 31 regroupent aussi une grande collection de tracts, et un ensemble de témoignages audio ;

- les archives du comité d'établissement (CE) d'Aerospatiale centralisent les procèsverbaux des séances plénières de l'institution (accès donné en 2012 par le secrétaire du CE pour la période 1945 à 1985, durant laquelle l'entreprise est une société nationale) ;

- les archives de l'Aérothèque de Toulouse, très riches d'un point de vue technique, apportent des informations économiques et sociales, en particulier autour des questions de l'évolution du travail (organisation, formation, qualification, etc.). Le fonds regroupe de manière quasi exhaustive nombre de publications d'entreprise (TO Infos, revue Aerospatiale, Courrier des cadres, etc.) ;

- des fonds d'archives complémentaires : archives personnelles de militants, presse, etc. ;

- des entretiens réalisés par l'auteur dans le cadre de son travail doctoral avec les acteurs de l'époque ;

- enfin, des témoignages et interviews conduits par des chercheurs ou étudiants à des époques différentes (des années 1980 à nos jours), et utilisés de façon secondaire. Ces documents peuvent prendre la forme d'enregistrements, de retranscriptions complètes ou encore de simples notes. Tous permettent, avec les sources écrites, une meilleure compréhension des enjeux de la période et de la mémoire qu'en gardent les acteurs. Au total, ce sont environ 80 témoignages qui ont été recueillis, dont 22 mobilisés directement pour cet article (9 CGT, 6 FO, 3 CFDT, 3 hiérarchie, 1 extérieur).

Dans une première partie, nous présentons les grands enjeux de la réforme des pratiques sociales qui se dessine dans l'entreprise à la fin des années 1970. Nous revenons ensuite de manière approfondie sur les stratégies directes mises en œuvre par la direction pour tenter d'influencer le déroulement de la rénovation sociale. Dans notre troisième partie, nous nous intéressons à des pratiques plus diffuses, que l'on peut rassembler sous le vocable de stratégies discriminatoires indirectes. 


\section{Contexte social et politique de la réforme engagée dans l'entreprise}

Durant la première moitié du $\mathrm{Xx}^{\mathrm{e}}$ siècle, le patronat de la métallurgie a essayé à différentes reprises de combattre le syndicalisme d'influence communiste (ou socialiste) dans les entreprises (FrABOULET, 2007). Dans l'aéronautique, après les grands affrontements des années 1930, l'entrée dans la guerre froide et les événements de mai-juin 1968, les années 1970 se traduisent par un regain d'activité des dirigeants pour reprendre en main les usines. Selon la CGT, dans les années 1960, « Marignane et Toulouse sont choisis [...] comme laboratoires de méthodes dont l'objectif est d'anesthésier le syndicalisme. [...] Elles sont le terrain où le syndicalisme, souvent uni, agit pour décrocher les avancées sociales » (BERNARD-RoIGT, 2010, p. 111).

\section{Une volonté de reprise en main des usines après une décennie très conflictuelle}

Dans les années 1950 et 1960, la conflictualité est très forte dans les usines de Sud-Aviation. Ainsi, le mouvement social se structure-t-il dans l'entreprise à l'échelon national suite à une succession de luttes (en 1960-1961, 1963 ou encore 1965). Une fragile unité syndicale permet d'obtenir des avancées en matière de salaires, de conditions de travail, etc. Dans un contexte où la collaboration européenne se renforce (de façon bilatérale avec le Concorde à partir de 1962, puis multilatérale avec l'A300 à la fin des années 1960), la répétition des conflits durs a des répercussions sur la production et sur la productivité du site toulousain. Des retards dans les livraisons et une dégradation de l'image de l'entreprise à l'échelle internationale en sont notamment des conséquences, selon la direction. Cette dernière souhaite donc réformer profondément les pratiques sociales à l'occasion de la fusion avec Nord-Aviation et la Société d'étude et de réalisation d'engins balistiques (Sereb). Un ancien de la CGT explique :

« Dans les années 1968, la direction a dit : "Il faut changer la politique sociale dans cette entreprise." Ils nous ont trouvés trop combatifs et peut-être trop efficaces dans la revendication. [...] On revendiquait une grille de salaires unique dans l'entreprise, mais la direction et la métallurgie n'en voulaient pas. Ils ont dit : "Il faut casser ça !" Ils ont cassé les salariés et surtout la CGT parce qu'elle était porteuse de ces revendications. »

(Archives M.-M. Rotelli et S. Rousseau, Interview de M. B. [CGT] ${ }^{7}$, retranscrit par M.-M. Rotelli et S. Rousseau, 29 mars 2013)

En 1967, le président-directeur-général (PDG) Maurice Papon ${ }^{8}$ nomme Fernand Carayon à la tête de l'établissement de Marignane. Celui-ci expérimente de nouvelles méthodes de gestion des ressources humaines dans ce bastion de la CGT et en réduit

7. Le sujet étant sensible et la plupart des acteurs encore en vie, les noms des personnes interrogées ont été « anonymisés ».

8. Ancien préfet de police de Paris, il est à l'époque connu pour avoir fait réprimer des mouvements sociaux (violences du métro Charonne en particulier), mais pas encore pour son rôle sous Vichy. 
fortement l'influence. Cet exemple, bien connu par ailleurs (MAHÉ, 2013), s'inscrit dans une démarche plus large de la direction cherchant à diminuer le poids du syndicalisme de tradition ouvrière et d'obédience communiste, très puissant au sein de l'industrie aéronautique française (CHAPMAN, 2011). Toulouse suit de près Marignane, dès le début des années 1970 ; St-Nazaire est le dernier affecté, à partir de la fin des années 1970, comme le rappellent récemment les militants de la CGT, d'abord dans un ouvrage (BELLIOT et al., 2012), puis dans un documentaire intitulé Le Laboratoire (MAHÉ, 2013).

Cette dynamique est une conséquence des fortes tensions sociales qui traversent l'entreprise (évolutions complexes du travail et des techniques, crise du travail, etc.). L'épisode de mai-juin 1968 apparaît décisif dans la volonté de réforme du dialogue social à Toulouse. Avec l'occupation de l'établissement et notamment du hall d'assemblage des prototypes, la direction locale prend conscience qu'il est indispensable de faire évoluer la régulation. À la différence de l'usine de Nantes-Bouguenais, où une section FO lambertiste est à la pointe du mouvement, précipitant le début de la grève avec occupation et séquestration de trois membres de la direction ${ }^{9}$, l'entrée en grève est assez tardive à Toulouse mais le mouvement est unitaire. Le prototype $001 \mathrm{du}$ Concorde est en cours d'assemblage, ce qui nécessite une continuité de l'activité sans quoi le prototype peut se dégrader rapidement et l'entreprise se retrouver en porte à faux vis-à-vis de ses partenaires et de ses assureurs. Les travailleurs ont ainsi un moyen de pression supplémentaire entre leurs mains, même s'ils ne l'utilisent à aucun moment : au contraire, les grévistes assurent les opérations de maintenance eux-mêmes et il ne leur est reproché aucune déprédation sur le prototype à la fin de l'occupation (JuILliet, 2015). Quoi qu'il en soit, la menace incite la direction à tout faire pour éviter à l'avenir les grèves à répétition ou les occupations d'usine, et pour cela à réduire l'influence du syndicalisme cégétiste et cédétiste ${ }^{10}$.

En 1969, avec la nomination de Jacques Chaban-Delmas comme Premier ministre, la direction s'appuie sur la dynamique nationale en cours pour tenter d'obtenir un apaisement au moyen d'une politique contractuelle et d'institutionnalisation du syndicalisme. Le projet de « Nouvelle société » vise précisément à favoriser le développement de la négociation collective et à dépasser les blocages de la société pour limiter le nombre de grèves. Si, dans la plupart des cas, cette tentative de réforme du dialogue social est un échec et aboutit à la poursuite d'une conflictualité intense (VIGNA, 2007), à Sud-Aviation Toulouse, c'est bien à une détente rapide que l'on assiste.

Après les grèves de mai-juin 1968, Maurice Papon est remplacé par Henri Ziegler. Proche de Chaban-Delmas depuis la Seconde Guerre mondiale, cet ancien chef d'étatmajor des Forces françaises de l'intérieur (FFI) a notamment été son directeur de cabinet au ministère des Travaux publics, des Transports et du Tourisme entre 1954 et 1956. Il préside l'une des composantes de l'Union des industries minières et de

9. À Toulouse, FO est très composite. Très modérée, elle rassemble des syndiqués de divers horizons autour d'un anticommunisme fédérateur.

10. Cela est aussi valable pour les sections politiques d'entreprise, au premier rang desquelles le Parti communiste français (PCF). 
la métallurgie (UIMM), l'Union syndicale des industries aéronautiques et spatiales, de 1971 à 1974 (SEIFFERT, 2010). Ensemble, ils souhaitent faire de la SNIAS l'un des laboratoires, l'une des vitrines et l'un des relais de la politique de réforme du dialogue social impulsée par le Premier ministre et par ses conseillers. Le premier d'entre eux, Jacques Delors, devient chargé de mission pour M. Chaban-Delmas. Il influence grandement sa politique en matière sociale entre 1969 et 1972, en particulier par les « contrats de progrès » et par l'importance accordée à la formation professionnelle. Les projets qui en découlent dans l'entreprise reposent en partie sur les réseaux politiques, économiques et sociaux et les relations qui peuvent se nouer entre les acteurs au plus haut niveau de l'État. Mais, pour les concrétiser, il leur est nécessaire de prendre appui localement sur un syndicalisme disposé à la négociation.

\section{Les années 1970 : une décennie charnière pour le syndicalisme}

Historiquement, l'industrie aéronautique constitue un important bastion du syndicalisme. Le site de Toulouse ne déroge pas à la règle. Dès avant la nationalisation de 19361937, des sections syndicales CGT ou CFTC et des sections politiques de gauche comme de droite sont présentes chez Latécoère et Dewoitine, qui formeront l'établissement de Toulouse. L'influence communiste est très prégnante à partir de la nationalisation de 1936-1937 et cette tradition se double du poids non moins important d'un syndicalisme réformiste, moins ouvrier et très anticommuniste, qui s'incarne dans la CGT-FO après la scission syndicale de 1947-1948 (DelPoux, 1990). Dans les années 1950, deux axes se constituent : l'un formé autour de la CGT et de la CFTC/CFDT soumet la possibilité d'engager une négociation au rapport de force préalable ; l'autre, qui rassemble FO et la CGC autour d'une conception réformiste et contractuelle, est plus disposé à la négociation $\mathrm{du}$ « grain à moudre » (DREYFus, PigENET, 2012), selon l'expression d'André Bergeron, secrétaire général de FO à de 1963 à 1989. Dans les années 1960, ces deux pôles sont capables de mener des actions communes et de remporter des victoires importantes. Mais, l'après mai-juin 1968 se traduit rapidement par une rupture de l'unité d'action syndicale.

Le déclin durable que connaît l'axe revendicatif peut s'expliquer de plusieurs manières, en particulier par la transformation socioprofessionnelle de la main-d'œuvre (LuCAS, 1989) et les restructurations productives (externalisation des tâches, évolution vers la conception et l'assemblage au détriment de la production, etc.) qui affectent l'entreprise (JALABERT, Zuliani, 2009). Mais il n'est sans doute pas indépendant des mesures répressives et des stratégies discriminatoires qui contribuent à une transformation profonde de l'établissement. Que cela soit dans le cadre des élections au CE (voir tableau 1) ou des élections des Délégués du personnel (DP, voir tableau 2), on constate une nette baisse de l'axe « contestataire », et plus particulièrement de la CGT. Le nombre des adhérents de la CGT diminue fortement, passant de 1347 en 1967 à 1082 en 1973 et 589 en $1979^{11}$.

11. CGT (sans date [1968]), Rapport financier, État des effectifs comparés, V ${ }^{e}$ Congrès des travailleurs horaires CGT ; CGT (1980), Industrie aéronautique et spatiale, supplément au Liaisons industries n $\mathrm{n}^{\mathrm{O}} 81,1^{\mathrm{er}}$ au 15 mai, p. 20. 
TABLEAU 1 - Évolution de l'influence syndicale au CE (1966-1986 ; tous collèges confondus)

\begin{tabular}{lccccc}
\hline & & \multicolumn{3}{c}{ En \% des suffrages exprimés } \\
\hline CGT & $\mathbf{1 9 6 6}$ & $\mathbf{1 9 7 2}$ & $\mathbf{1 9 7 6}$ & $\mathbf{1 9 8 2}$ & $\mathbf{1 9 8 6}$ \\
CFDT & 50,0 & 38 & 37,7 & 26,0 & 20,7 \\
FO & 17,2 & 16 & 13,6 & 23,6 & 15,7 \\
CGC & 25,3 & {$[46$} & {$[48,7$} & 50,4 & 63,6 \\
CFTC & 7,3 & $\mathrm{X}$ & $\mathrm{X}$ & & \\
\hline
\end{tabular}

Source : calcul de l'auteur à partir des archives de l'IDHS CGT 31.

TABLEAU 2 - Évolution de l'influence syndicale au sein des DP (1966-1986 ; tous collèges confondus)

\begin{tabular}{lccccc}
\hline & & \multicolumn{3}{c}{ En \% des suffrages exprimés } \\
\hline CGT & $\mathbf{1 9 6 6}$ & $\mathbf{1 9 7 2}$ & $\mathbf{1 9 7 6}$ & $\mathbf{1 9 8 2}$ & $\mathbf{1 9 8 6}$ \\
CFDT & 49,7 & 38,1 & 37,8 & 24,7 & 20,5 \\
FO & 17,1 & 14,9 & 12,3 & 24,4 & 14,2 \\
CGC & 24,8 & 38,3 & 37,5 & 34,4 & 42,8 \\
CFTC & 8,4 & 8,7 & 8,9 & 10,9 & 16,1 \\
\hline
\end{tabular}

Source : calcul de l'auteur à partir des archives de l'IDHS CGT 31.

Le 3 septembre 1970, un millier de grévistes manifestent sur le tarmac proche de l'usine de Saint-Martin-du-Touch, à l'occasion de la présentation de la Caravelle 12 et du prototype Concorde 001 à « la presse et aux personnalités du monde aéronautique et politique ${ }^{12}$ ». La cérémonie doit représenter, selon M. Ziegler, un « coup d'éclat » visant à soutenir une relance de l'entreprise ${ }^{13}$. Si cette démonstration de force, qui selon certains militants se tient dans un « climat insurrectionnel ${ }^{14} »$, peut apparaitre anecdotique au premier abord dans le contexte de l'après mai-juin 1968 et n'a pas d'impact visible à court terme, elle marque un tournant majeur. Considérée par la direction comme une action malveillante, une « odieuse manifestation » dans un contexte économique et productif morose, la décision de perturber l'événement marque une inflexion très nette des rapports sociaux. Selon un membre de la hiérarchie :

« CGT et CFDT envahissent la cérémonie, renversent les tables et foutent tout en l'air et Dufour [directeur de l'établissement de Toulouse], dont on a dû vous dire qu'il avait une âme de combattant [...], dit : "Vous voulez la guerre, ben vous allez l'avoir" et de là naît une partition entre le syndicalisme dur et le syndicalisme plus réformiste. Finalement, CGT et CFDT finissent dans l'opposition après la perte du CE et trouvent en face d'eux "une direction forte", qui ne leur pardonne pas l'événement.

12. Mirassou J. (1970), « Caravelle 12-Airbus-Concorde » et « Manifestation des travailleurs de l'Aerospatiale pendant la présentation des avions », La Dépêche du Midi, Édition Toulouse, 4 septembre.

13. CE de la SNIAS Toulouse, Assemblée plénière extraordinaire, $\mathrm{n}^{\circ} 236,21$ août 1970.

14. Archives P. Chaskiel, Notes de P. Chaskiel sur l'interview de R. P. (CFDT), $1^{\mathrm{er}}$ août 2002. 
Cela m'a été raconté aussi bien par Dufour que par Bertholio [chef du personnel].

[...] Cela avait quand même fait scandale. »

(Interview de J.-M. F. [hiérarchie] par l'auteur, 5 novembre 2014)

Si la portée du geste est réelle, son caractère symbolique témoigne également d'un accroissement des tensions dans l'établissement. Cette inflexion ne cible qu'une partie des organisations, en priorité la CGT et la CFDT, alors même que les tenants de l'axe réformiste ont participé à la manifestation et se sont montrés menaçants lors de l'assemblée extraordinaire du CE du 21 août 1970, qui avait pour but d'informer les syndicats de la tenue de la cérémonie. La création de la SNIAS cette même année entérine une rupture syndicale qui va structurer le mouvement social pendant plusieurs décennies. Il s'agit d'un cas original de reprise en main des usines au moyen d'une réforme des pratiques s'appuyant sur un syndicalisme réformiste influent et sur un ensemble de stratégies qui s'apparentent à des procédés discriminatoires, dans un contexte où les syndicats de l'axe contestataire seraient, selon les militants interrogés, affaiblis depuis $1968^{15}$.

\section{Les stratégies directes}

La direction s'appuie sur plusieurs leviers pour faire évoluer les pratiques sociales : des discriminations salariales aux blocages de carrières, en passant par le contrôle de l'embauche et la mobilisation de la maîtrise et des cadres.

\section{Les discriminations salariales et blocages de carrières}

Dans les années 1960, les augmentations individuelles de salaire (AIS) et les reclassements sont proposés tous les six mois (avril et octobre) à une partie des salariés, selon des critères de mérite et d'ancienneté, dans le cadre d'un budget prédéfini. Ce sont les agents de maîtrise et les cadres qui en ont la responsabilité dans leur service. Avec la signature de l'accord d'entreprise en octobre 1970, les augmentations collectives sont négociées annuellement dans le cadre d'une procédure définie qui permet une « garantie de progression des niveaux de vie et des rémunérations ${ }^{16}$ » et contient une clause d'augmentation automatique semestrielle de $2 \%$ (indexée sur l'inflation jusqu'en juin $1986^{17}$ ). Plusieurs militants CGT et CFDT expliquent qu'une fois l'accord signé, il fallait avoir « la bonne carte syndicale » pour bénéficier d'une augmentation individuelle, avoir une promotion ou être reclassé ${ }^{18}$. Certains militants

15. Archives P. Chaskiel, Notes de P. Chaskiel sur l'interview de G. C. (CGT), 21 février 2002.

16. SNIAS, Accord d'entreprise personnels ouvrier, mensualisé et mensuel non cadre, Paris, 21 octobre 1970.

17. L'accord est dénoncé par la direction en juin 1986. Elle ne souhaite plus garantir une augmentation automatique des salaires dans le contexte d'une nouvelle politique de maîtrise des coûts salariaux.

18. Archives P. Chaskiel, Notes de P. Chaskiel sur l'interview de L. G. (CFDT), 25 juillet 2002 ; Archives P. Chaskiel, Notes de P. Chaskiel sur l'interview de G. C. (CGT), 21 février 2002. 
de FO sont, semble-t-il aussi, bloqués dans leur carrière, l'un d'entre eux affirmant avoir bénéficié d'un « avancement minimum ${ }^{19}{ }$. Dès le mois de février 1972, la CGT dénonce la nécessité, dans certains secteurs de l'établissement, d'adhérer à un syndicat plutôt qu'à un autre pour être sûr d'en bénéficier régulièrement, ce qui instaure à sons sens un « climat malsain ${ }^{20} »$ dans les usines.

Selon elle, l'attribution des augmentations doit être collective et automatique selon le principe « à travail égal, salaire égal » et non reposer sur le mérite individuel et le service rendu à l'entreprise, comme ce serait alors le cas ${ }^{21}$. En octobre 1972, la CGT de l'usine de Blagnac affirme que ses militants ont été « fusillés » et «puni[s] » en raison des journées d'action menées en juin 1972 pour le maintien de leur pouvoir d'achat « parce qu'ils sont à la CGT ou [...] à la CFDT », ce qu'elle considère comme une discrimination. D'après le récit qui en est fait dans un tract adressé aux cadres et agents de maîtrise, les participants auraient été « rayés » des listes des AIS, pratique qui va «à l'encontre du droit de grève » et qui « remet en cause les libertés fondamentales ${ }^{22} »$. La direction ne prend pas seulement en compte les « aptitudes professionnelles », mais bien des critères politiques et syndicaux, même en cas de rapport favorable de la maîtrise, ce qui contribue à entretenir « une ambiance et un climat de crainte ${ }^{23} \gg$. Donnant quelques exemples, la section cégétiste note que certains n'ont pas bénéficié de reclassements depuis trois à six ans ${ }^{24}$.

Mais les tracts et dénonciations syndicales n'ont pas permis de faire cesser ces pratiques, qui ne sont condamnées, et par-là même juridiquement attestées, que plus de trente ans plus tard. Ainsi, en 2006, un ancien de la CGT remporte son procès en appel pour « discrimination syndicale ${ }^{25} »$. Dans l'extrait du jugement, il est démontré qu'embauché en 1968, il a été promu en 1971 et 1974, mais ne l'a plus été jusqu'en 1998. Selon la CGT, à l'usine St-Martin, « la Direction ne pren[d] en compte que les demandes d'augmentation et de promotion des sympathisants ou adhérents à une organisation signataire » de l'accord d'entreprise ${ }^{26}$. Plus largement, la question de la répartition des augmentations salariales a fait l'objet de plusieurs procédures depuis 1998 et notamment de procès remportés par 330 militants et retraités des établissements de Nantes, Saint-Nazaire, Méaulte et Toulouse ${ }^{27}$. Depuis les années 1970, la plupart d'entre eux ont vu leur carrière bloquée en raison de leur appartenance syndicale ou

19. Archives P. Chaskiel, Notes de P. Chaskiel sur l'interview de L. C. (FO), 29 juillet 2002.

20. CGT, «AIS », Le Point de vue, 9 février 1972.

21. CGT, La Direction mène le jeu, 19 avril 1972.

22. CGT, Aux cadres et agents de maîtrise, 11 avril 1972.

23. Interview de G. D. (CGT) et A. M. (CGT) par l'auteur, 10 mars 2015.

24. CGT, « Encore des discriminations », Le Proto, 6 octobre 1972.

25. Cour d'appel de Paris, $18^{\mathrm{e}}$ chambre, section A, Arrêt du 21 mars 2006, RG n ${ }^{\circ}$ 04/35526.

26. CGT, Discriminations, 10 avril 1972.

27. Voir par exemple, Cour d'appel de Paris, $18^{e}$ chambre, section D., Arrêt du 9 mars 2004, $\mathrm{n}^{\circ} 13$, extrait des minutes du Greffe, 9 mars 2004 ; Cour de Cassation, Chambre sociale, Pourvoi n ${ }^{\circ}$ R 04-42.293, Décision n ${ }^{\circ} 11431$ F, 8 juin 2005. 
de leurs opinions politiques ${ }^{28}$. Suite à la condamnation définitive de l'entreprise en cassation, près de 30 millions d'euros leur auraient été accordés au total en réparation ${ }^{29}$.

De fait, tout au long des années 1970 et 1980, on trouve de nombreux témoignages et traces écrites dénonçant, ou parfois simplement relatant, les blocages de carrière et refus d'augmentation que subissent certains militants syndicaux. À côté des tracts soulignant les situations de discrimination ou de « ségrégation ${ }^{30}$ », le problème est dénoncé durant l'été 1975 lorsque la CFDT porte plainte contre la direction ${ }^{31}$. En effet, en septembre 1974, dans un contexte de baisse des plans de charge, des déplacements de personnels vers Marignane, qualifiés d'autoritaires par certains syndicats, engendrent un dur conflit. Il se termine par la « séquestration » de trois membres de la hiérarchie et une intervention des forces de l'ordre. Tous les participants CGT et CFDT voient leurs reclassements supprimés pour fait de grève, ce qu'ils dénoncent comme relevant d'une discrimination salariale ${ }^{32}$. Au mois de mai 1976, alors qu'un procès pour séquestration vise les militants syndicaux, un tract mentionne l'inculpation du chef du personnel et du directeur pour atteinte à la liberté syndicale. De fait, le procureur demande une peine d'amende pour « discrimination concernant les délégués syndicaux », comme le relate la presse de l'époque ${ }^{33}$. La CFDT rappelle qu'à Marignane ou à Châteauroux, des affaires sont en cours pour ces raisons, ce qui indique que la stratégie semble se déployer dans l'ensemble de l'entreprise ${ }^{34}$.

La Dépêche du Midi publie, dans le cadre de son enquête réalisée à la suite de la publication du tract par la CFDT le 25 août 1981, plusieurs témoignages anonymes révélant un contrôle de l'attribution des promotions en fonction de l'appartenance syndicale, ce que conteste en réponse la direction ${ }^{35}$. La CFDT accompagne ses propos du témoignage d'un chef de service qui raconte : « Nous établissons des propositions à la direction. Après être passées par divers échelons, [elles] nous reviennent. Et j' ai pu constater que, pour certains, existe un véritable veto de la part de la direction ${ }^{36}$. » Un deuxième confirme ces pratiques : « On regarde [...] si les gens proposés ont fait grève, s'ils sont engagés politiquement, syndicalement. Et on fait le tri. Mais, en tant que chef de service, on nous oblige à "couvrir" les décisions de veto prises par la direction ${ }^{37}$ ».

\footnotetext{
28. Cette thématique mériterait une étude systématique à partir de l'évolution des salaires et de l'ensemble des carrières sur un échantillon large de salariés.

29. Interview de G. D. et A. M. (CGT) par l'auteur, 10 mars 2015 et université populaire de Toulouse, IDHS 31, Diffusion/Débat autour du film Le Laboratoire, 24 et 25 février 2015. Voir aussi les nombreux articles consacrés à la question.

30. CGT, Communiqué du Cartel SNIAS CGT, Paris, 27 avril 1971.

31. CFDT, « Des directeurs de la SNIAS devant les juges », Flashs de rentrée, 13 août 1975.

32. Ibid.

33. « Second procès. Mêmes acteurs mais rôles inversés », La Dépêche du Midi, édition Haute-Garonne, 7 décembre 1978.

34. CFDT, La Direction de la SNIAS inculpée, 25 mai 1976.

35. «Les coulisses de l'emploi : La direction : "nous accordons une importance particulière à la sélection" », La Dépêche du Midi, Édition Haute-Garonne, 15 septembre 1981.

36. Ibid.

37. «Les coulisses de l'emploi : La direction : "nous accordons une importance particulière à la sélection" », art. cité.
} 
Un ancien militant de la CGT revient aussi sur ces pratiques :

« J'étais complètement bloqué. [...] Mon chef d'équipe, qui était un délégué de la CGC avec qui j'ai toujours eu de bons rapports [...], faisait des propositions de promotions pour son équipe et il m'avait mis en tête de liste. Quand il a porté la liste au contremaître, il lui a dit : "Il te faut la refaire cette liste !" ; alors mon chef d'équipe m'a mis en troisième position et le contremaître lui a refait la même réflexion jusqu'à ce qu'il m'ait enlevé de la liste. Ce n'est pas le contremaître qui m'enlevait, c'était mon copain, le chef d'équipe et ça l'embêtait ».

(Archives M.-M. Rotelli et S. Rousseau, Retranscription de l'interview de M. B. [CGT] par M.-M. Rotelli et S. Rousseau, 29 mars 2013)

En septembre 1983, M. Barnet, inspecteur du travail, réalise à la demande de la CGT, une étude sur l'évolution des rémunérations des représentants syndicaux ${ }^{38}$. Dans le compte rendu qu'il adresse au syndicat, il rappelle qu'indépendamment de tous les constats confidentiels qui peuvent être dressés sur les individus, leurs mandats, etc., l'attribution des augmentations individuelles les dix années précédentes démontre « une différence globale très nette entre [...] les représentants des organisations FO et CFTC et ceux des organisations CGT et CFDT ». Pour les représentants ouvriers (premier collège), $80 \%$ des CFDT et $90 \%$ des CGT ont moins d'une augmentation tous les dix-huit mois, alors que les taux sont respectivement de 15 et $20 \%$ pour la CFTC et FO. Le même constat, « légèrement atténué », est dressé pour les employés, techniciens et agents de maîtrise (deuxième collège). En ce qui concerne le salaire, « l'analyse de la situation révèle $[\ldots]$ une nette différence » puisque $30 \%$ des syndicalistes de FO ont une rémunération supérieure de $500 \mathrm{~F}^{39}$ à la moyenne de la catégorie concernée alors que c'est seulement le cas de $8 \%$ pour ceux de la CFDT et d'aucun pour ceux de la CGT. L'inspecteur conclut ainsi sa lettre : «Compte tenu de tous ces éléments de fait, j'ai confirmé à la direction de votre établissement la nécessité de prendre toute disposition pour redresser les anomalies constatées en suggérant d'employer la voie de la négociation. » En 1989, dans l'étude sur les trajectoires professionnelles suivies par les salariés dans le contexte des évolutions techniques observées entre 1945 et 1985 , qu'il mène en coopération avec des sociologues de l'université Toulouse-Le Mirail, Michael KLEIN (1989) observe des différences dans les carrières de vingt salariés de l'entreprise (de divers métiers et catégories professionnelles). Parmi les cinq facteurs qui contribuent à les expliquer, sont notamment évoqués « le syndicalisme et la politique sociale de l'entreprise ». Il l'indique explicitement (p. 348) :

«La carrière peut être accélérée pour les membres d'un syndicat, et freinée pour les membres d'un autre; et ce rapport peut s'inverser d'une époque historique à une autre. La politique sociale peut soutenir ou contrecarrer les stratégies syndicales, contrôler les embauches et les promotions internes selon ses propres critères. »

38. Barnet J.-P., Lettre au secrétaire du syndicat CGT de la SNIAS de la part de l'IT, 27 septembre 1983.

39. Soit environ 150 euros en 2015. 
Pour qualifier les évolutions de carrières, il parle de « carrières ouvertes » et de « carrières fermées » et affirme (p. 352 et 353) :

«Avant 1970, la tradition de promotions par la CGT était bien établie ; après, [les] promotions se faisaient avec l'aide de FO. [...] Mais ceci reste un phénomène pas quantifiable. Par contre, le freinage de carrières pour les syndiqués CGT ou CFDT à partir de 1972 est un phénomène généralement reconnu, même par les membres des autres syndicats [...]. Au niveau hiérarchique, là où se jouent les possibilités de promotions et de carrières, l'alliance FO et CGC avec la direction de Toulouse avait trois résultats essentiels : FO devenait l'interlocuteur privilégié de la direction, ce qui assurait un avancement hiérarchique pour ses membres ; les membres et les sympathisants de la CGT et de la CFDT furent écartés de tous les postes de maîtrise et de commandement ; les embauches furent de plus en plus contrôlées par les syndicats dits "coopératifs", afin de marginaliser tout syndicalisme militant. »

Michael KLEIN note en outre que des membres de la CFDT ont pris leur carte chez FO pour obtenir de l'avancement. D'autres témoignages indiquent des mouvements similaires à la CGT : un cégétiste affirme ainsi que «beaucoup de militants CGT passent à $\mathrm{FO}^{40}$ », ce qui peut expliquer en partie la forte baisse du nombre d'adhérents que connaît le syndicat.

\section{Contrôle de l'embauche}

Il est très difficile de prouver la discrimination à l'embauche, car l'employeur peut le plus souvent présenter des motifs dits « objectifs » pour appuyer ses refus (GARNERMOYER, 2003). Un certain nombre d'indices permettent toutefois de s'interroger sur d'éventuelles discriminations alors même que la situation de l'entreprise est difficile d'un point de vue économique : les embauches sont bloquées entre 1973 et 1979, et la direction fait appel à une main-d'œuvre d'appoint pour répondre aux fluctuations d'activité.

En avril 1971, la CGT dénonce une discrimination en matière d'embauche, qui s'opérerait par le recours à des sociétés de location de personnel, en particulier la Compagnie de méthodes et de gestion $(\mathrm{CMG})^{41}$. Cette entreprise de travail temporaire se charge de répondre aux demandes à la place de la SNIAS. Le préambule du contrat de travail pour la CMG stipule ainsi : « [...] suite à votre demande d'emploi, nous vous offrons de vous embaucher dans notre établissement de Toulouse, sous réserve de l'accord de l'autorité de tutelle [de la SNIAS]. » Aucune durée n'y est mentionnée et le contrat est une simple feuille volante ${ }^{42}$. La CFDT affirme que l'embauche n'est plus directe et que le personnel doit faire un stage dans une entreprise extérieure, qui

40. Archives P. Chaskiel, Notes de P. Chaskiel sur l'interview de G. C. (CGT), 21 février 2002.

41. CGT, Le Bulletin du syndiqué, 17 août 1971.

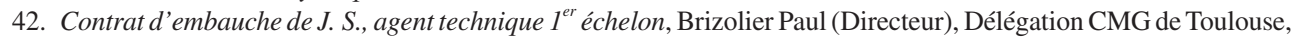
2 août 1971. 
le loue ensuite à la SNIAS ${ }^{43}$. Les syndicats, peu préparés à ce type d'emploi sous statut différent et non-encadré ${ }^{44}$, l'assimilent à une sélection déguisée des salariés. La CFDT souligne que les idées des futurs embauchés peuvent être contrôlées et que les travailleurs ainsi recrutés sont lésés puisqu'ils ne font pas directement partie de l'entreprise (conditions salariales, de congés, etc., différentes) ${ }^{45}$. La CGT déclare que s'il n'y a pas de durée d'embauche, c'est bien pour pouvoir licencier « plus facilement, du jour au lendemain sans trop de remous », tout en pratiquant un «filtrage de l'embauche ${ }^{46}$ ». $\mathrm{Si}$, pour la CGT, l'opération grève les budgets de l'entreprise et « se double d'une opération discriminatoire courante ${ }^{47} »$, pour la direction, la CMG est avant tout un intermédiaire administratif ${ }^{48}$ qui aide à pallier les difficultés économiques ${ }^{49}$. Selon un militant de FO, l'embauche est bien sélective, mais elle n'a pas de visée discriminante : «Il s'agit d'avoir des gens fiables pour construire des avions ${ }^{50}$. »

D'ailleurs, après plusieurs interventions orales en ce sens, FO et la CGC demandent formellement en mai 1972 à la direction l'embauche des personnels sous statut CMG, considérant que les « délais prévoyant leur adaptation, se trouvent largement dépassés ${ }^{51}$ ». À cette même période, la CFDT veut l'arrêt de ce type « [d'] embauche scandaleuse $^{52}$ » et la fin du « grenouillage » dans les usines. Pour elle, « les organisations "officielles" (FO et CGC) semblent découvrir l'existence de la CMG. Car, depuis le début, jamais FO et CGC ne sont intervenus à ce sujet. Le mot "CMG" n'est pas même apparu dans un seul de leur tract ou dans une seule de leurs interventions ${ }^{53}$ ". Pourtant, dès le 11 janvier 1972, FO s'est déclaré contre le recours à un «négrier »et pour l'embauche définitive des travailleurs en contrat avec la CMG qui ont « atteint les trois mois d'essai ${ }^{54} »$. Le 16 juin 1972, elle revendique avoir obtenu le recrutement de 214 travailleurs $^{55}$, ce qui semble bien indiquer que la direction accorde une attention particulière aux demandes de ce syndicat.

Selon un militant de la CGT, certains membres de FO se rendent auprès des intérimaires pour leur signaler que s'ils veulent être embauchés, il est préférable qu'ils adhèrent à $\mathrm{FO}^{56}$. Il ne s'agit pas ici de mettre en cause ce syndicat, mais plutôt de décrire un changement de paradigme et de pratiques dans l'établissement et, plus largement, dans l'entreprise. En effet, dans d'autres secteurs industriels comme les mines,

\footnotetext{
43. CFDT, Pourquoi de nouvelles méthodes d'embauche, 18 août 1971.

44. Il faut attendre la loi du 3 janvier 1972 (loi 1972-1) pour que le recours à l'intérim soit codifié.

45. CFDT, Pourquoi de nouvelles méthodes d'embauche, doc. cité.

46. CGT, Le Scandale de l'embauche, 18 août 1971.

47. CGT, Déclaration des élus et représentants CGT à M. le Préfet de région, août 1972.

48. Interview de J.-M. F. (hiérarchie) par l'auteur, 5 novembre 2014.

49. CE de la SNIAS Toulouse, Assemblée plénière, nº 249, 16 octobre 1972.

50. Interview de B. M. (FO) par l'auteur, 21 novembre 2013.

51. FO, CGC, Information au personnel, 18 mai 1972.

52. CFDT, L'Embauche scandaleuse par la CMG cessera-t-elle bientôt ?, mai 1972.

53. Ibid.

54. FO, Information au personnel, 11 janvier 1972.

55. FO, Informations sociales, 16 juin 1972.

56. Archives P. Chaskiel, Notes de P. Chaskiel sur l'interview de R. R. (CGT), 19 février 2002.
} 
le papier-carton ou les docks, la CGT a pu bénéficier de pratiques similaires. Quoi qu'il en soit, l'inflexion est sensible à Toulouse, puisque pour être embauché dans les années 1940 et 1950, il était souvent nécessaire de passer soit par le PCF et la CGT, soit par l'évêché comme l'affirment certains acteurs ${ }^{57}$. Trente ans plus tard, au milieu des années 1970, la situation est tout autre. Recruté à cette période comme négociateur de contrats commerciaux, un sympathisant de la CGT témoigne :

«Quand je suis arrivé, j’étais militant communiste. [...] J'avais fait très attention pour que ça ne soit pas su par la direction. Je n'étais pas kamikaze. Au bout de trois-quatre ans, dans les années 1978-1979, lorsque je commençais à être connu et que je savais que mes patrons m'appréciaient, j'ai accepté de figurer dans une liste électorale communiste avec ma tête sur les affiches à Colomiers. J'étais allé voir mon patron et puis je lui avais dit : "Vous savez, j' ai un problème un peu particulier, les communistes de Colomiers m'ont demandé de figurer sur une liste électorale avec eux". [...] Je lui ai demandé ce qu'il en pensait sur le fond ; il a dit : "Je suis prêt à en discuter quand vous voulez." [...] Il me dit : "Donnez-moi deux-trois jours pour réfléchir car c'est quand même pas courant comme façon de faire." Au bout de deux jours, il m'appelle et me dit : "Écoutez, j'en ai parlé au siège, si vous ne changez pas votre comportement au bureau, je ne vois pas ce que je peux dire, mais je tiens à vous prévenir que quand même, dans le contexte de l'Aerospatiale [SNIAS], il vaut mieux renoncer à toute promotion". »

Il ajoute :

« Je ne pense pas que ça ait facilité ma carrière. [...] Mais je ne mélangeais pas les deux. En dépit des avertissements, ça ne m'a pas empêché de finir cadre supérieur pendant les quinze dernières années de ma vie professionnelle. [...] D'une certaine manière, je n'étais pas dangereux, j'étais trop isolé. Donc [...]j'étais un peu la caution de tolérance dont on pouvait parler pour dire qu'il n'y avait pas de répression. »

(M.-M. Rotelli, S. Rousseau, Retranscription de l'interview de J. L. [CGT] par M.-M. Rotelli et S. Rousseau, le 24 janvier 2013)

Les 26 et 27 janvier 1981, le député communiste M. Montdargent attire l'attention du ministre des Transports (M. Hoeffel) et du ministre du Travail (M. Mattéoli) sur les graves atteintes aux libertés syndicales constatées à la SNIAS de Toulouse ${ }^{58}$. Quelques mois plus tard, M. Bapt, député socialiste, adresse un courrier au nouveau ministre du Travail (M. Auroux) dans lequel il lui fait part du mauvais climat social régnant dans l'entreprise ${ }^{59}$. Il joint le témoignage d'un ancien stagiaire du service du personnel, M. Lafontan, initialement révélé par la CFDT dans un long tract quelques jours plus tôt, le 25 août 1981 :

« Je suis entré officiellement à l'Aerospatiale [SNIAS] le lundi 11 juin 1979. [...]

Il fallait $[\ldots]$ instaurer un filtrage très serré, au niveau du recrutement, pour éviter

57. Voir notamment : Archives P. Chaskiel, Notes de P. Chaskiel sur l'interview de J. T. et J.-P. N. (FO), 7 mars 2002.

58. Assemblée nationale, Questions et réponses, $n^{\circ} 41721$ et 41722, 26 janvier 1981.

59. G. Bapt, Lettre à M. Jean Auroux, Paris, 7 septembre 1981. Je n'ai pas connaissance d'une réponse du ministre du Travail. 
de faire rentrer à l'Aerospatiale, non seulement des militants, mais toute personne susceptible de voter CGT ou CFDT. [...] En ce qui concernait l'embauche des techniciens, tâche qui m'incombait, je devais effectuer les filtrages à partir des dossiers et des entretiens. Pour cela, j'allais être aidé par les délégués FO, car ils venaient souvent au service du personnel recommander des candidats. »

(CFDT, Les Libertés à la SNIAS-Toulouse, 25 août 1981)

Ce stagiaire démissionne la semaine suivante. Dans son communiqué en réponse à la CFDT, la direction fait part de son étonnement et dénonce un coup monté. Elle rappelle que la politique sociale s'appuie sur deux piliers, l'efficacité et l'aspiration individuelle, et repose, en matière de sélection, sur des critères de qualité et de motivation $^{60}$. Dans sa réponse à la direction, la CFDT donne la parole au «chef de la sécurité » - sans le nommer clairement - de l'établissement de Saint-Nazaire (19711981), qui évoque le même type d' « actions anti-syndicales ${ }^{61} »$.

\section{Mobilisation de la maîtrise et des cadres}

La discrimination syndicale passe aussi par une mobilisation de la maîtrise et des cadres. Par certains aspects, elle peut devenir un outil de management. Elle a pour corollaire la mise à l'écart de ceux qui ne s'inscrivent pas dans ses orientations. Encore une fois, Marignane constitue un laboratoire puisque le directeur M. Carayon y met en place un système de mise à l'écart très poussé, qui se généralise dans les années suivantes (MAHÉ, 2013). Il s'agirait notamment, au moyen de stages et de réunions de s'assurer de l'implication de la maîtrise et des cadres à qui serait inculquée une communauté d'intérêts. Pour la CGT, il faut « obtenir que les cadres mettent à la disposition de leur employeur non seulement leur expérience et leurs connaissances professionnelles, mais [aussi] leurs pensées et convictions ${ }^{62} »$.

Entre 1946 et 1965, le directeur local toulousain, M. Grimaud, n'avait pas instauré de politique particulière en matière de recrutement des maîtrises et des cadres, qui étaient souvent issus des rangs ouvriers et de la promotion interne. Dans les années 1970, un changement complet est opéré, puisque son successeur à ce poste, M. Dufour, recourt à l'embauche de salariés extérieurs ${ }^{63}$ pour accompagner la conversion des agents de maîtrise en managers alors plus soucieux, d'après la CGT, « d'orienter, de diriger, de canaliser, de sélectionner les individus suivant leurs opinions syndicales ou philosophiques » que d'encadrer leur travail ${ }^{64}$. Pour le syndicat, cette sélection traduit une volonté « d'épuration » très nette qui vise à briser les mouvements revendicatifs. Sûre de sa force au tout début des années 1970, l'organisation affirme que cette stratégie

\footnotetext{
60. «Les coulisses de l'emploi : La direction : "Nous accordons une importance particulière à la sélection" », La Dépêche du Midi, Édition Haute-Garonne, 15 septembre 1981.

61. CFDT, Information au personnel, 24 septembre 1981.

62. CGT, Discriminations, 10 avril 1972.

63. Archives P. Chaskiel, Notes sur l'interview d'H. G. (CGT), 9 février 2002.

64. CGT, Les Agents de maîtrise, 19 avril 1971.
} 
antigrève est mise en échec et ne contribue qu'à désorganiser la production dans certains ateliers $^{65}$. Toutefois, quelques années plus tard, dans un tract daté du 4 avril 1978, elle dénonce la mutation autoritaire d'un chef d'équipe en poste depuis seize ans, écarté peu de temps après avoir suivi un stage de la Société d'organisation et de gestion industrielle et commerciale (Sogic, cf. infra). Selon le syndicat, il a pourtant « démontré ses grandes capacités et [...] toute sa conscience professionnelle au service de l'entreprise ». Il serait le onzième à être congédié en raison de son "étiquette syndicale », sans jamais que la direction de l'établissement fournisse la moindre explication $^{66}$.

La CGT et la CFDT dénoncent ces convocations de maîtrises et de cadres par leur hiérarchie. Selon les deux syndicats, « les plus avertis ${ }^{67}$, sont éliminés, chassés », « on pousse à la retraite les vieux cadres moins maniables, et on parachute des "jeunes loups" dont l'habileté à résoudre les problèmes se mesure à la longueur de leurs dents $^{68}{ }^{\star}$. Toujours d'après eux, ces pratiques entraînent des tensions dans les ateliers ; il n'est « plus tenu compte de la qualité professionnelle » mais bien des accointances d'opinion $^{69}$. Un ancien animateur de ce type de formations donne sa version des faits sur son blog, à la tonalité politique très marquée :

«À cette époque, je travaillais comme chef du personnel puis directeur des relations humaines au sein du groupe Pierre Fabre. Je n'avais pas trop mal réussi dans ce métier passionnant. Aussi étais-je souvent demandé pour animer, à la lumière de mon expérience et de mes connaissances des idéologies, des journées de formation pour des cadres et agents de maîtrise, et aussi pour des syndicalistes non inféodés au Parti communiste. Pierre Fabre m'accorda alors de pouvoir m'absenter systématiquement un jour par semaine pour ce projet de bon fonctionnement hiérarchique et de paix sociale dans les entreprises. Bertholio [chef du personnel de l'établissement toulousain de la SNIAS] s'occupait de recruter les stagiaires, notamment des militants syndicalistes alors très anticommunistes [de FO], de la CFTC et de la CGC. C'est ainsi que par-delà nos divergences, $\mathrm{j}$ 'eus le grand plaisir de rencontres fréquentes avec ce grand honnête homme qu'était André Bergeron, le "patron" de FO. Très malicieux, il me disait avec un bon sourire : "On n'est pas de la même paroisse, mais je préfère envoyer mes gars entendre vos sermons plutôt que celui des cosaques." Les "cosaques" dans le parler syndical, c'était la CGT ; c'est-à-dire les rouges, les Soviétiques. Et mes "sermons", c'était toute une formation en six journées sur six semaines à la contre-subversion, une sorte de vaccination contre les virus bolcheviques et gauchistes ${ }^{70} »$.

65. CGT, « La nouvelle société dans nos ateliers », Le Hublot, septembre 1970.

66. CGT, « Un chef d'équipe relevé de ses fonctions de commandement », Le Point de vue, 4 avril 1978.

67. Par ce terme, comprendre « ceux qui n'adhèrent pas aux visées de la direction ».

68. CGT, CFDT, Ce que cache la concertation au niveau de la maîtrise, 25 février 1971.

69. CGT, À quand les lettres de cachet ?, 4 mars 1971.

70. Anthony B. (2014), « Retour sur la "dénationalisation" de l'aéroport de Toulouse », Le Blog de Bernard Antony, 9 décembre. En ligne : http://www.bernard-antony.com/2014/12/retour-sur-la-denationalisation-de.html ; consulté le 25 août 2016. 
Dans la deuxième moitié des années 1970, ces stages « s'appuient notamment sur la Sogic, entreprise également utilisée à Saint-Nazaire pour former les maîtrises et cadres à la gestion du personnel ». Un classeur ${ }^{71}$ que nous avons pu consulter relate le contenu des cours qui y sont dispensés : physionomie, psychologie, communication, sociologie, etc. Selon la direction, elle ne fait appel aux services de la Sogic que pour détecter les potentiels et apprendre aux cadres à développer "une meilleure communication avec les hommes $^{72} »$. Mais pour un membre de la CGT, ces stages sont « idéologiques »; ils visent à inculquer un « esprit maison » et une implication sans faille au service des objectifs définis par la direction ${ }^{73}$.

\section{Les stratégies indirectes}

La signature de l'accord d'entreprise en 1970 représente un premier moyen de division syndicale. La direction favorise par ce biais l'axe réformiste et contribue à faire basculer le CE à son profit. Cette démarche s'accompagne de nouvelles pratiques mises en place pour réduire l'influence du syndicalisme CGT et CFDT.

\section{La signature de l'accord société}

La fusion des sociétés nationales de construction aéronautiques aboutit en 1970 à la création de la SNIAS dans le cadre d'un processus de concentration et de modernisation impulsé quelques années plus tôt par Georges Pompidou. Cette évolution impose l'unification des conditions salariales et sociales des 43000 salariés. Aussi, des négociations se tiennent-elles dès le premier trimestre cette année-là entre la direction et les syndicats CGT, CFDT, FO, CFTC, CGC, unis autour d'une plate-forme de revendications, malgré les désaccords qui peuvent exister entre les organisations. La concertation sociale traduit en partie la volonté d'instaurer une « nouvelle société », selon l'expression du Premier ministre Jacques Chaban-Delmas, de modernisation sociétale. Si la demande d'un accord d'entreprise est une revendication très ancienne dans les entreprises constitutives de la SNIAS ${ }^{74}$, elle est constamment repoussée jusqu'en 1967, à la différence de ce qui a pu se passer dès les années 1950 dans d'autres entreprises pionnières, Renault en tête (KARILA-COHEN, WILFERT, 1998).

Sans entrer dans le détail du processus qui conduit à la signature de l'accord, les syndicats, confrontés à « la lenteur, l'inefficacité des négociations et l'insuffisance des propositions ${ }^{75} »$, décident de lancer un mouvement de grève dès le retour

71. Voir IDHS 31. Classeur Sogic, Formation des agents de maîtrise Aerospatiale, Cycle de cours 1977-1978.

72. Affaires sociales Aerospatiale Toulouse, « Si nous parlions Formation », Le Bulletin, n 14 novembre 1980.

73. Archives P. Chaskiel, Notes de P. Chaskiel sur l'interview d'H. G. (CGT), 9 février 2002.

74. Elle est exprimée pour la première fois dès 1955-1956 par un syndicat indépendant dans le cas de Toulouse.

75. CFDT, Ah! C'est donc cela la concertation. Négociation et répression dans une société nationale, Livre blanc publié par l'Inter-CFDT de la société Aerospatiale, novembre 1970. 
des congés d'été. Mais, après l'envahissement de la cérémonie du 3 septembre 1970 (cf. supra), la direction parvient à provoquer une rupture de l'unité d'action syndicale et à obtenir une signature séparée de FO, la CGC et la CFTC. Ainsi, l'accord relatif aux non-cadres ${ }^{76}$ est-il signé le 21 octobre 1970, dans un contexte de très fortes tensions inter-syndicales, qui débouchent dès le lendemain sur des affrontements et, à l'échelle de l'établissement, une rupture définitive (DELPECH, 2010). Selon plusieurs acteurs de l'établissement, en échange de leur signature, la direction aurait promis aux syndicats réformistes de les aider à conquérir le $\mathrm{CE}$ aux élections suivantes ${ }^{77}$.

Ce sont principalement le préambule, l'article 3 et l'article 5 qui posent problème à la CGT et à la CFDT. Le texte stipule :

« [Préambule] Les parties signataires,

- soucieuses de garantir, d'une part, un développement du pouvoir d'achat, d'autre part, un développement économique favorable à la fois à la politique d'emploi et à la politique de progrès ;

- convaincues de la nécessité du dialogue entre les représentants de la Direction et ceux du Personnel, en vue d'atteindre dans de meilleures conditions les objectifs ci-dessus ; s'engagent à tout mettre en œuvre pour y parvenir et à ne pas remettre en cause les sujets traités dans le cadre de l'accord, pendant la durée de son application. [...]

Article 3 : Adhésions ultérieures : le présent accord constitue un tout indivisible, tant dans son esprit que dans sa lettre ; l'adhésion ultérieure d'une organisation syndicale non-signataire ne peut être partielle et doit donc porter sur l'accord dans son entier. [...]

Article 5 : Procédure de conciliation : [...] Jusqu'à l'expiration des délais [...] les parties contractantes s'engagent à ne susciter aucune autre forme d'action, qui pourrait être provoquée par les difficultés ayant donné lieu aux procédures ci-dessus. » (SNIAS, Accord d'entreprise personnels ouvrier, mensualisé et mensuel non-cadre,

21 octobre 1970, Paris, p. 1 et 2)

L'accord entérine un principe de négociation exclusive entre la direction et les signataires, favorisant par là une division syndicale. Pour la CGT, « au niveau de la Société, la [direction générale] s'appu[ie] sur deux organisations minoritaires [et prend] la responsabilité, malgré ses engagements, d'exclure des discussions [sur l'accord] la CGT et la CFDT représentant la majorité du personnel SNIAS ${ }^{78} »$. Le texte conventionnel, pierre angulaire du dispositif stratégique d'ensemble, s'accompagne de fait d'un isolement des non-signataires par le biais d'une réforme beaucoup plus large des pratiques sociales en matière d'institutions représentatives (cf. infra). La direction considère nécessaire de faire place à un syndicalisme plus disposé à discuter de revendications sur les bases définies par elle, tout en montrant qu'il y a plus à gagner par la concertation que par la contestation. Selon un ancien de FO, il s'agit

76. L'accord relatif aux cadres est paraphé par toutes les organisations en juillet 1970.

77. Archives P. Chaskiel, Notes de P. Chaskiel sur l'interview de G. C. (CGT), 21 février 2002. Archives P. Chaskiel, Notes de P. Chaskiel sur l'interview de B. D. et A. E. (hiérarchie), 27 juin 2002.

78. CGT, Au mépris de la démocratie, la CGT écartée de la gestion du CE, 8 mars 1972. 
« de signer un pacte avec la direction, qui s'appelle l'[accord société], de dire on en a ras le bol de faire grève pour n'importe quoi et de perdre de l'argent, on veut revenir dans la négociation collective. C'est vraiment le pacte que nous avait demandé de faire Bergeron qui disait que si 10 sous sont gagnés, c'est déjà 10 sous de gagnés qui ne sont plus à prendre ».

(Interview de B. M. [FO] par l'auteur, 21 novembre 2013)

Selon les différents retraités que nous avons interrogés, les rangs des non-signataires $^{79}$ sont divisés sur la stratégie à suivre. Certains pensent qu'il faut signer pour ne pas se trouver en dehors de l'espace laissé à la négociation et ne pas pratiquer une politique de la chaise vide ; d'autres estiment à l'inverse qu'avec un tel accord, ils vont se retrouver pieds et mains liés. Tous s'accordent cependant aujourd'hui à reconnaître que ne pas le parapher fut une erreur qui a permis à la direction de poursuivre sa stratégie, alors qu'une signature n'aurait engagé à rien. Dès le 9 mai 1972, un accord conçu selon le même modèle est signé à la Société nationale d'étude et de construction de moteurs d'aviation [Snecma] par les syndicats, y compris la $\mathrm{CGT}^{80}$. À l'Aerospatiale [SNIAS], ce syndicat signe finalement l'AS le 21 septembre 1972, car il a compris que sans cela, aucun espace ne lui était laissé pour développer ses idées. Mais la roue a déjà tourné, puisque l'organisation syndicale poursuit son déclin. La CFDT ne le signe que le 29 décembre 1981, après l'accession de la gauche au pouvoir, malgré les fortes tensions qui la parcourent tout au long des années 1970 et alors même qu' elle augmente ses scores entre 1976 et 1982 , au fur et à mesure de son recentrage ${ }^{81}$, avant de décliner définitivement au profit de FO et de la CFTC, recréée en 1973 dans l'établissement.

\section{Le basculement du CE}

Le second versant de cette stratégie de rénovation du dialogue social s'incarne dans le renversement du CE en 1972. Alors qu'il était géré depuis le milieu des années 1960 au prorata de l'influence syndicale (malgré des tensions qui pouvaient intervenir entre les tenants des deux axes, contestataires et réformistes), et sur une base revendicative pour la biennale de $1970^{82}$, le scrutin du 10 février 1972 marque une évolution très nette. À cette occasion, FO et la CGC s'allient et forment une liste appelée «L'entente », qui vise à conquérir le $\mathrm{CE}$ pour le gérer de manière « homogène », c'est-à-dire sans la participation de la CGT ou de la CFDT. La principale raison qui motive cette initiative est un désaccord sur ce que doit être l'institution : avant tout organisme de lutte des classes pour la CGT et la CFDT, il est un lieu privilégié d'information économique et de gestion des œuvres sociales au service de tous pour la FO et la CGC. Les résultats

\footnotetext{
79. Témoignages de MM. B., C., R., L., G., etc.

80. FO, Information au personnel, $1^{\mathrm{er}}$ juin 1972.

81. Pour un aperçu général des questions posées par le recentrage de la CFDT au tournant des années 1980, on se reportera à DEFAUD (2009). Des investigations complémentaires doivent être menées pour l'expliquer dans l'établissement toulousain.

82. Cf. CE de la SNIAS-Toulouse, Assemblée plénière, n $^{\circ} 234,24$ avril 1970. Le mandat de gestion du CE est de deux ans.
} 
de l'élection sont équilibrés (voir tableau 1) : «L'entente » ne totalise que $46 \%$ des suffrages, dans un contexte où la direction a pourtant participé de manière très active à la campagne. Elle a pris parti de diverses manières : par une absence de neutralité et la mobilisation de la hiérarchie ${ }^{83}$, par l'aide au recueil du suffrage des personnels en congés longue maladie ${ }^{84}$, par le vote enfin en faveur de cette liste (lors de l'élection du bureau ${ }^{85}$ ) qui, sans cela, n'aurait pas été en mesure de gérer seule le CE et aurait dû parvenir à un compromis avec les autres listes ${ }^{86}$. L'élection enregistre un record de participation avec $90,1 \%$, dans un contexte où il y a près de 1000 inscrits de plus qu'en 1970. On compte près de $7 \%$ de plus de votants dans le premier et le deuxième collèges et près de $17 \%$ dans le troisième collège ce qui, comme le souligne la CGT, « dénote une subite prise de conscience ${ }^{87}$ » des ingénieurs et cadres. La CGT fait remarquer que la direction a lancé une « offensive idéologique » visant à réduire son influence et celle de la CFDT « dans le but d'intégrer les travailleurs aux objectifs de l'entreprise ${ }^{88} »$. Elle dénonce en particulier « les avantages outranciers accordés à FO

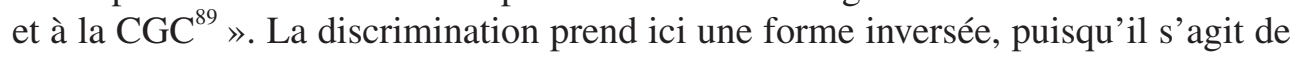
promouvoir un syndicalisme au détriment d'un autre, par exemple en votant lors de l'attribution des responsabilités au CE (bureau, commissions, délégations). Malgré une majorité relative, «L'entente » gère seule le CE dans les années 1970, et au-delà. Signe, là aussi, d'une stratégie nationale de la part de la direction, une alliance semblable prend le contrôle des CE d'autres établissements entre 1972 et 1978 (Marignane, Cannes, Bourges) et la direction du comité central d'entreprise (CCE) en 1979.

FO et la CGC notent « que des divergences fondamentales [...] s'opposent à tout compromis pour une gestion saine et correcte » du CE avec les autres organisations syndicales. Pour elles, il s'agit d'assurer dans cette instance « une représentation homogène » et « au service de tous », alors que la CGT était présentée jusque-là comme pratiquant une politique de discrimination envers les syndiqués du bloc réformiste en matière d'œuvres sociales ${ }^{90}$, critique récurrente formulée dans les années 1950 et $1960^{91}$. Aussi ces organisations proposent-elles un bureau composé de membres FO et CGC, des commissions avec vice-présidence alternée entre les deux syndicats et des délégations dans les institutions des œuvres sociales (centre culturel, centre

83. CGT, « Pour cadrer les cadres », Le Point de vue, février 1972.

84. Archives P. Chaskiel, Notes de P. Chaskiel sur l'interview d'Y. M. (CFDT), 16 juillet 2002.

85. Le Président du CE, qui est le directeur de l'établissement ou son représentant, dispose d'une voix tandis que les représentants titulaires des salariés élus disposent des dix autres voix. À l'occasion de l'élection de 1972, si «L'entente » ne rassemble pas $50 \%$ des voix, elle compte toutefois cinq sièges, grâce à l'application du quotient électoral, contre quatre à la CGT et un à la CFDT. Il faut souligner par ailleurs le glissement d'un siège du premier vers le deuxième collège à l'occasion de ce scrutin, qui bénéficie à « L'entente », puisque celle-ci domine nettement les deuxième et troisième collèges (respectivement employés, techniciens, agents de maîtrise d'une part ; et ingénieurs et cadres, d'autre part).

86. CGT, Après les élections du CE, 15 février 1972.

87. CGT, Bulletin du syndiqué, 24 février 1972.

88. CGT, Examen de la situation à la SNIAS-Toulouse, Enseignement des élections, mars 1972.

89. Ibid.

90. Interview de B. M. (FO) par l'auteur, 21 novembre 2013.

91. FO et CGC, Déclaration, 8 mars 1972. Interview de G. R. et C. V. (FO) par l'auteur, 7 novembre 2013. 
sportif, coopérative et mutuelle) exclusivement issues de «L'entente ». Selon la CGT et la CFDT, deux blocs se font désormais face et cette décision « impérialiste » accentue la division des travailleurs : CGT et CFDT d'une part, FO, CGC et direction d'autre $\operatorname{part}^{92}$.

\section{De nouvelles pratiques de régulation sociale}

D'autres leviers sont enfin utilisés pour isoler les syndicats et les empêcher d'influencer la régulation sociale. Par exemple, les DP ne sont plus réunis mensuellement comme le veut la loi, mais à intervalles beaucoup plus irréguliers. En 1972, la CGT doit ainsi entreprendre par deux fois des démarches auprès de l'inspection du travail pour faire respecter le droit ${ }^{93}$. En outre, les syndicats constitutifs de «L'entente » font le choix de ne plus participer aux réunions DP-direction, puisque les revendications sont désormais traitées dans le cadre de l'accord d'entreprise. Ils préfèrent demander un rendez-vous à la direction pour aborder les cas individuels. De fait, tout au long des années 1970, on ne compte que peu de réunions des DP et celles-ci basculent très rapidement dans un dialogue de sourds, les syndicats rappelant inlassablement leurs revendications et la direction se contentant de réponses lapidaires dans des assemblées de très longue durée (14 heures en trois jours pour celle de mars 1972 par exemple).

Après la fusion de 1970, les DP subissent aussi la concurrence d'un nouvel acteur dans la régulation sociale : le correspondant social. Celui-ci doit contribuer à établir un lien direct entre la direction et les travailleurs au sein d'îlots puis de groupes opérationnels décentralisés, créés dans les années $1970-1980^{94}$ : l'opération vise à rapprocher les salariés de leur hiérarchie, à les rassembler en plus petits groupes, afin de pouvoir mieux prendre en compte leurs aspirations individuelles, sans passer par un intermédiaire, ou tout du moins en passant par un intermédiaire hiérarchique plutôt que syndical. En 1972, la CGT dénonce sa « haute-main » en matière d'embauche, de mutation ${ }^{95}$ voire de déroulement de carrière ${ }^{96}$. Elle dresse un bilan de cette « appellation [qui] ne figure pas au catalogue des professions ». Si elle reconnaît que la fonction vise à faciliter l'intégration du personnel et à l'aider « à surmonter les difficultés qui ne pourraient être résolues par la hiérarchie », la CGT affirme qu'il s'agit aussi de lui faire accomplir un certain nombre de « sombres besognes ${ }^{97}$ » et de court-circuiter l'action syndicale.

La direction impose par ailleurs un encadrement plus strict de l'espace usinier, afin de faire baisser les contacts entre les travailleurs et les militants. Les plus combatifs et les plus prosélytes font l'objet d'un isolement géographique au sein d'un atelier de

92. CFDT, Le Nouveau CE, 8 mars 1972.

93. CGT, Délégués du personnel, réunion mensuelle, 24 mars 1972.

94. Archives P. Chaskiel, Notes de P. Chaskiel sur l'interview de M. B. (CGT), 21 juin 2001.

95. CGT, « Scandale et mépris », Le Proto, 4 janvier 1972.

96. CGT, CFDT, Non à l'arbitraire, 19 janvier 1972.

97. CGT, « Rallonges et reclassements », Le Rapido, n 67, 8 mai 1973. 
l'usine St-Eloi, l'atelier Bobillot, qualifié de « goulag $^{98}$ » par un membre de la CGT. L'équivalent marignanais est la « mine de sel » (hangar Boussiron), implantée à près de trois kilomètres de l'usine principale ${ }^{99}$. Parfois, on ne confie aucun travail à ces salariés. Ils se contentent de rester assis et/ou de lire la presse ${ }^{100}:$ «On prenait soixante-dix travailleurs de la CGT, on les mettait dans un même endroit. Ainsi, les travailleurs n'avaient personne à convaincre ${ }^{101}$. » On observe une dévalorisation du travail de ces militants, dont les tâches ne sont souvent plus en rapport avec leurs compétences ${ }^{102}$. Ces méthodes s'accompagnent d'un développement de la communication interne dans les années 1970, qui vise à concurrencer la parole syndicale et à la noyer dans un flot de publications. Peut-être, faut-il y voir le pendant, dans l'entreprise, de la création d'une commission de l'information par Georges Glasser au sein du CNPF (VIGNA, ZANCARINI-FOURNEL, 2010).

Le dernier moyen privilégié que l'on peut noter à l'encontre de la CGT et de la CFDT est une stricte application du règlement intérieur. Les sanctions révèlent des motifs divers : absence de signature de bon de délégation, pointage réalisé par un autre, distribution de tracts, prises de parole, etc. Jusqu'à la fin des années 1960, il n'y a pas, semble-t-il par tradition, de contrôle précis de l'utilisation des heures de délégation par les représentants du personnel. Cela change dès le début de la décennie suivante. Si le contingent accordé est de 1600 heures par mois tous représentants confondus, près de 5000 heures par mois seraient prises par les délégués dans le cadre de leurs mandats. Aussi, afin de réduire ce nombre, la direction prend la décision de facturer au $\mathrm{CE}$ et aux syndicats les heures de dépassement. Certains retraités affirment que cette mesure ne concerne pas les militants de «L'entente» qui seraient, parfois, en délégation permanente ${ }^{103}$. Une note de service d'avril 1972 précise : «Si vous êtes amené à dépasser ce temps [...], il vous sera retenu sur votre salaire en fin de mois ${ }^{104}$. » Au regard de la documentation consultée, il semble néanmoins peu probable que la menace ait été mise à exécution.

Ces éléments de contrôle pris ensemble démontrent très nettement une volonté de la direction d'entraver l'expression syndicale par une répression qui s'abat de manière différente selon l'appartenance syndicale. Elle s'apparente à de la discrimination, sans qu'il soit possible d'en évaluer la portée réelle, mais les documents et témoignages rassemblés ici ne laissent que peu de doutes.

\footnotetext{
98. Interview d'E. P. (CGT) par l'auteur, 3 décembre 2014.

99. Archives P. Chaskiel, Notes de P. Chaskiel sur l'interview de B. D. et A. E. (hiérarchie), 27 juin 2002.

100. Entretien de l'auteur avec Y. C. (CGT).

101. Archives M.-M. Rotelli et S. Rousseau, Retranscription de l'interview d'Y. C. (CGT), 18 octobre 2012.

102. Interview de G. D. et A. M. (CGT) par l'auteur, 10 mars 2015.

103. Interview d'E. P. (CGT) par l'auteur, 3 décembre 2014.

104. Bertholio E. (1972), Note de service : heures de délégation, Aerospatiale, usines de Toulouse, $\mathrm{SP}, \mathrm{n}^{\circ}$ 531.725/72,

EB/HT/MF, 28 avril.
} 
Comme le détaille la note de la direction générale rédigée en 1978 (BELlot et al., 2012 ; cf. supra), à partir de cette date, il s'agit de renforcer la communication et de « faire apparaître que [les] organisations syndicales [réformistes, à savoir FO, la CGC et la CFTC] obtiennent des résultats ». Il faut leur accorder des entrevues dans les meilleurs délais quand elles le demandent et faciliter leur circulation dans l'usine par l'attribution de "postes faciles, sans tâches [...] ou non chronométrés, hors chaînes, ou encore des postes avec contact avec le personnel (caristes, magasiniers, service d'entretien, animateurs de prévention, contrôleurs volants, ou autres postes à haut degré de liberté $)^{105} \gg$. En outre, la hiérarchie doit repérer les travailleurs qui pourraient se présenter sur les listes réformistes en respectant certains critères : « Forts en gueule, meneurs, apt[es] au commandement, influents sur les compagnons de travail (y compris sur les femmes et les immigrés), motivés politiquement (Association ouvrière professionnelle, Rassemblement pour la République), anticommunistes, anciens militaires ou gendarmes, personnes ayant des problèmes personnels importants (famille, santé, diplôme, salaire, promotion) » et expliquer au « candidat potentiel [...] l'intérêt qu'il peut avoir à agir, soit pour lui (besoin de considération, avantage direct, salaire, promotion, etc.), soit pour la collectivité (résultats obtenus) ». Il faut enfin lui dire « que seuls les trois syndicats susnommés lui permettront d'accéder à ce but ».

La direction s'appuie ainsi sur une large palette d'outils pour tenter de résorber l'influence d'un type de syndicalisme au profit d'un autre, plus gestionnaire et plus à même d'assurer et de garantir une certaine paix sociale, pratique qui n'a pas disparu aujourd'hui (PENISSAT, 2013, comme plus largement les articles de ce numéro). Sont utilisées à cette fin des stratégies indirectes et collectives, qui viennent compléter les stratégies directes et individuelles. Mais les premières, à la différence des secondes, restent encore peu connues des sciences sociales (droit ou économie), notamment car, au premier abord, la notion de discrimination s'appuie principalement sur la reconnaissance de cas particuliers et non sur un ensemble de pratiques plurielles et plus générales, mais aussi plus voilées.

Les matériaux utilisés permettent de revenir plus en détail sur les mécanismes, les ressorts, les visées ou encore les conséquences que peuvent avoir ces méthodes qui ont sans nul doute participé d'une baisse durable de l'influence des syndicats CGT et CFDT à court, moyen et long terme. À court terme, cette « réforme sociale » a pour conséquence principale de provoquer un raidissement du pôle contestataire, aussi bien dans l'établissement de Toulouse que plus largement dans l'entreprise, en particulier à St-Nazaire à la fin des années 1970. Durant la décennie suivante, le management participatif, au travers de la démarche Innovation, dynamisme économique, expression des salariés (Idées), et l'utilisation de nouveaux outils (comités d'ateliers et de bureaux, boîtes à idées, cercles de qualité et groupes de pilotage, etc.) complètent les dispositifs pour isoler la CGT dont la position au sein de l'établissement est de plus en plus difficile. Mais ils ont aussi désormais des conséquences sur la place laissée à un

105. « Note très confidentielle - SNIAS 1978 », cité dans BELLIOT et al. (2012), pp. 54-56. 
syndicalisme réformiste, qui n'adhère pas à cette nouvelle phase de réforme sociale. Celui-ci est en effet concurrencé dans ses canaux d'expression par un lien direct établi entre la hiérarchie et les travailleurs au moyen de ces nouvelles institutions, qui visent à renforcer la communauté d'intérêts entre salariés et direction autour d'objectifs communs. À moyen et long termes, c'est bien à une perte d'influence durable du syndicalisme cégétiste et cédétiste que l'on assiste. Bien tardifs, les procès intentés et remportés par les salariés victimes de tout ou partie de ces faits de discrimination corroborent nos hypothèses de départ, mais ne permettent pas de remonter le temps.

\section{BibliograPHIE}

Belliot G., Chevalier D., Le Lan C., Lemasson D., Mahé M., Patillon C. (récit mis en forme par) (2012), Debout et libres! La CGT dans l'aéronautique nazairienne (1923-2010), Nantes, Éditions du Centre d'histoire du travail.

BERnARD-RoIGT M. (coord.) (2010), L'Aéronautique : une histoire sociale en Midi-Pyrénées, Toulouse, Institut régional d'histoire sociale CGT Midi-Pyrénées.

CARLIER C. (1983), L’Aéronautique française (1945-1975), Panazol, Lavauzelle.

CHAPMAN H. (2011), L'Aéronautique : salariés et patrons d'une industrie française (19281950), Rennes, Presses universitaires de Rennes.

DEFAUD N. (2009), La CFDT, 1968-1995. De l'autogestion au syndicalisme de proposition, Paris, Presses de la Fondation nationale des sciences politiques.

DELPECH V. (2010), La Vie syndicale dans le département de la Haute-Garonne de 1967 à 1978, mémoire de Master II d'histoire contemporaine, Toulouse, Université de Toulouse-Le Mirail.

DeLPoux Y. (1990), Étude sur la scission de la CGT et la naissance de la CGT Force ouvrière en Haute-Garonne : janvier 1936-décembre 1948, thèse de $3^{\mathrm{e}}$ cycle en histoire, Toulouse, Université de Toulouse-Le Mirail.

Dreyfus M., Pigenet M. (dir.) (2012), Les Meuniers du social : Force ouvrière, acteur de la vie contractuelle et du paritarisme, fin des années cinquante-début des années quatre-vingt, Paris, Publications de la Sorbonne.

FRABOULET D. (2007), Quand les patrons s'organisent. Stratégies et pratiques de l'Union des industries métallurgiques et minières, 1901-1950, Villeneuve d'Ascq, Presses universitaires du Septentrion.

GARNer-Moyer H. (2003), « Discrimination et emploi : revue de la littérature », Document d'étude, $\mathrm{n}^{\circ} 69$, Paris, Direction de l'animation de la recherche, des études et des statistiques (Dares).

Jalabert G., Zuliani J.-M. (2009), Toulouse : l'avion et la ville, Toulouse, Privat.

Juilliet C. (2015), «Occuper une usine de haute technologie : Sud-Aviation Toulouse en mai-juin 1968 », Annales du Midi. Revue de la France méridionale, n² 289, pp. 83-104. 
KARILA-COHEn P., WiLfert B. (1998), Leçon d'histoire sur le syndicalisme en France, Paris, Presses universitaires de France.

Kechidi M., Tадвот D. (2006), «L'industrie aéronautique et spatiale : logique d'arsenal, logique commerciale », in Colletis G., Lung Y., La France industrielle en question. Analyses sectorielles, Paris, La Documentation Française, pp. 78-86.

KLEIN M. (1989), Trajectoires professionnelles dans l'industrie aéronautique, Toulouse, M. Klein [Auto-édition, polycopié]. Voir résumé : http://pmb.cereq.fr/index.php?lvl=author_ see\&id=4819; consulté le 7 septembre 2016.

LuCAs Y. (dir.) (1989), Le Vol du savoir. Techniciens de l'aéronautique et évolution des technologies, Lille, Presses universitaires de Lille.

MahÉ N. (2013), Le Laboratoire, Crossac [France], Les films du Grippaud, 71 minutes.

Mercat-Bruns M. (2011), « La discrimination », in Bevort A., Jobert A., Lallement M., Mias A., Dictionnaire du travail, Paris, Presses universitaires de France, p. 184.

MoREL C. (1994), La Grève froide : stratégies syndicales et pouvoir patronal, Toulouse, Octarès éditions.

Penissat É. (dir.) (2013), « Réprimer et domestiquer : stratégies patronales », Agone, $\mathrm{n}^{\circ} 50$.

Rotelli M.-M., Rousseau S. (2014), L'Évolution du travail dans l'industrie aéronautique du début des années 1960 au début des années 1980, master II en histoire contemporaine, université de Toulouse-Le Mirail.

SeIFFERT M.-D., « Henri Ziegler », in Daumas J.-C. (dir.) (2010), Dictionnaire historique des patrons français, Paris, Flammarion, pp. 725-727.

Vigna X. (2007), L'Insubordination ouvrière dans les années 68 : essai d'histoire politique des usines, Rennes, Presses universitaires de Rennes.

Vigna X., Zancarini-Fournel M., « Mai 1968 », in Daumas J.-C. (dir.) (2010), Dictionnaire historique des patrons français, Paris, Flammarion, pp. 1335-1339. 